\title{
Metastatic follicular thyroid carcinoma mimicking sacroiliitis
}

\author{
Sasan Fallahi \\ Internist, Rheumatologist, assistant professor of Tehran University of Medical Scienecs, Internal Medicine Division, Baharloo \\ Hospital, Behdari Street, South Kargar Street, Tehran, Iran; Rheumatology Research Center, Tehran University of Medical Sciences, \\ Tehran, Iran
}

\begin{abstract}
Sacroiliac joint involvement is not specific to seronegative spondyloarthropathies ( $\mathrm{SpA})$. There are some rare reports of conditions which mimic sacroiliitis. This paper reports a case of follicular thyroid carcinoma in a patient who had been referred to a rheumatology clinic with hip pain. Sacroiliac joint involvement was her presenting feature before she was correctly diagnosed. The patient was a female, 63 years of age, who referred to a rheumatologist because of unilateral hip pain. Patrick's test on her right side was positive, and the range of motion of her right hip was restricted. Imaging studies included conventional hip radiography and magnetic resonance imaging (MRI) revealed sacroiliitis with surrounding destructive lesion. In review of systems and physical examination, a thyroid nodule was found. Computed tomography (CT)guided needle biopsy of her hip and fine needle aspiration (FNA) of the thyroid nodule established follicular thyroid carcinoma. In approach to sacroiliitis, rare differential diagnoses (besides $\mathrm{SpA}$ ) and mimickers of sacroiliitis such as neoplastic lesions should also be considered to avoid misdiagnosis.
\end{abstract}

Keywords: magnetic resonance imaging, radiography, sacroiliitis, thyroid carcinoma.

\section{Introduction}

Hip pain due to sacroiliitis is the main symptom of ankylosing spondylitis (AS). However, sacroiliac joint involvement is not exclusive to spondyloarthropathies $(\mathrm{SpA})$, and there are several other conditions which may mimic sacroiliitis. Several such mimickers have been reported, including brucellosis, gluteal abscess, pyogenic sacroiliitis, tuberculosis, Ewing sarcoma, epithelioid sarcoma, osteoid osteoma, sarcoidosis, metastatic adenocarcinoma, hyperparathyroidism, osteomalacia, osteitis condensans ilii, and sacral insufficiency fracture [1-11]. Herein, a case of thyroid cancer who referred to a rheumatology clinic due to hip pain is reported. Sacroiliac involvement was the chief complaint before proper diagnosis.

\section{Case presentation}

A 63-year-old female referred to the rheumatology clinic of Baharloo Hospital, Tehran University of Medical Sciences with pain in the right hip without fever from 3 months prior to her referral. The patient's past medical history was unremarkable with the exception of a thyroid nodule 5 years ago. Physical examination revealed range of motion in the right hip joint was restricted. Patrick's test suggested the sacroiliac joint as the source of hip pain. No peripheral arthritis in other joints was detected. Spinal movements were normal in forward flexion, extension, right and left lateral bending. Physical examination revealed a nodule (about $3.5 \mathrm{~cm}$ ) in the right lobe of the thyroid. The results of laboratory analysis parameters, i.e. complete blood count (CBC), erythrocyte sedimentation rate (ESR), C-reactive protein (CRP), Wright, Coombs-Wright, and tuberculin skin test, serum level of calcium, phosphorus, and alkaline phosphatase, were unremarkable. The radiographic study of the hip was suggestive of sacroiliac involvement with a destructive lesion in the right sacroiliac region (Fig. 1). The coxofemoral joint was intact without abnormality. Naproxen 1000 mg/day was initiated, but pain was not significantly reduced. An MRI was requested, which revealed a destructive lesion on the right side of $\mathrm{S} 1$ of the sacrum extending to the sacroiliac joint with edema of the iliacus and gluteal muscles and hypersignality in bone marrow, more in favor of a malignant tumor (Fig. 2). Ultrasonography of the thyroid gland revealed a $3.5 \mathrm{~cm}$ nodule in the right lobe with calcified wall. Cytology obtained by fine needle aspiration (FNA) of the thyroid nodule was compatible with follicular neoplasm. 


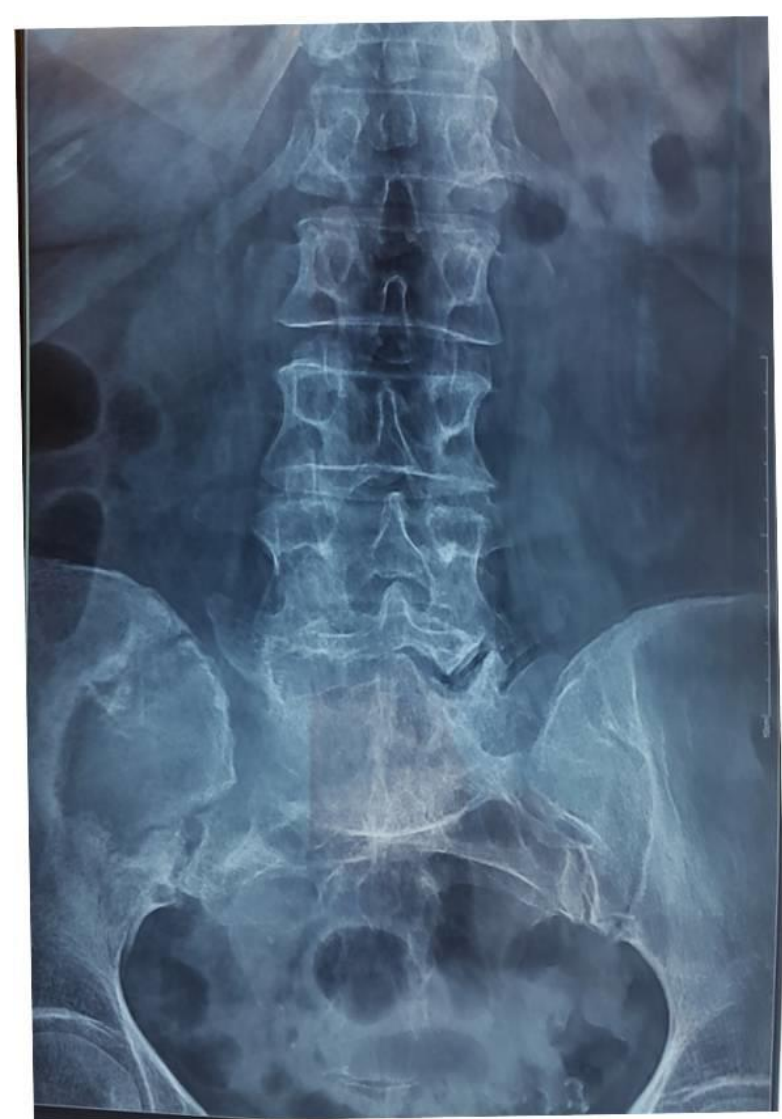

Fig. 1. Hip radiography

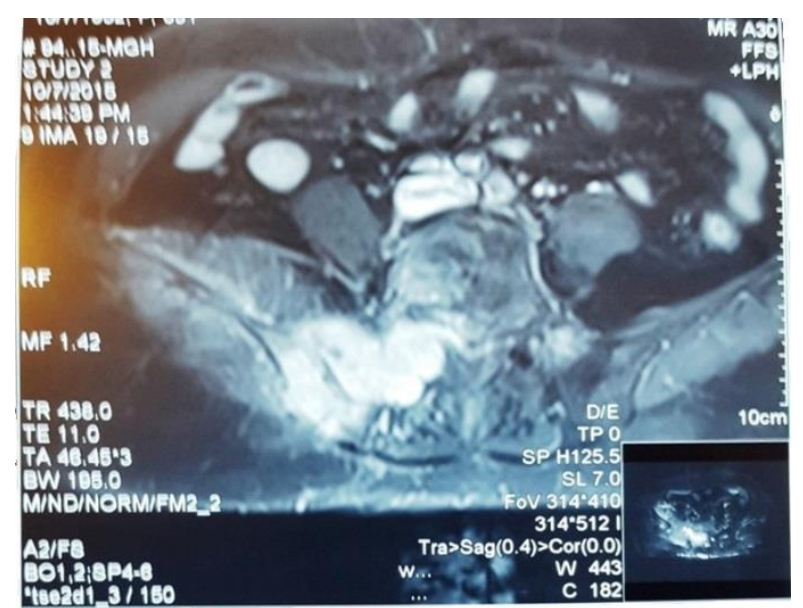

Fig. 2. MRI of hip
The patient submitted to a CT-guided needle biopsy of bone mass in her right pelvic region performed by an interventional radiologist. Pathological examination established the diagnosis of metastatic follicular thyroid carcinoma. The patient was referred to an endocrinologist and an oncologist for therapeutic managements.

\section{Discussion}

Follicular cancer of the thyroid is the second most common type of thyroid cancer. This carcinoma metastasizes through the hematogenous route. Metastases occur in 10-15\% of cases. The most common site of distant metastasis is bone (with a lytic pattern). Hip involvement has been reported in 5-13\% of bone metastases [12]. To the best of the authors' knowledge, this is the first report of follicular cancer of the thyroid as a mimicker of sacroiliitis. In this case, the clinical and imaging clues in support of malignant neoplasm rather than rheumatologic manifestation (seronegative spondyloarthropathy) were as follows: hip pain with no response to anti-inflammatory dose of non-steroidal anti-inflammatory drug, persistent severe hip pain throughout the day without morning stiffness or exacerbation at morning, unilateral involvement of the sacroiliac region without other peripheral and axial joint involvement, discovery of a large thyroid nodule through physical examination of all body systems in addition to the musculoskeletal system, and destructive and lytic pattern of sacroiliac joint involvement in conventional radiography.

\section{Conclusion}

$\mathrm{AS}$ and other seronegative SpA are the most common causes of sacroiliitis; however, other rare differential diagnoses such as neoplasm should be kept in mind. Awareness and attention to clinical clues can prevent faulty or delayed diagnoses.

\section{Conflicts of interest}

The author declares no conflicts of interest.

\section{References}

1. Slobodin G, Boulman N, Rimar D, Kaly L, Rozenbaum M, Rosner I, et al. Acute sacroiliitis. Clin Rheumatol 2016; 35(4): 851-6. doi: 10.1007/s10067-0163200-6.

2. Gómez Rodríguez N, Peteiro
Cancelo A, Ibáñez Ruán J, González Pérez M. Epithelioid sarcoma of the right ilium mimicking sacroiliitis. Reumatol Clin 2013; 9(2): 120-2. doi: 10.1016/j.reuma.2012.03.008.

3. Binicier O, Sari I, Sen G, Onen F,

\author{
Akkoc N, Manisali M, et al. Axial \\ sarcoidosis mimicking \\ radiographic sacroiliitis. \\ Rheumatol Int 2009; 29(3): 343- \\ 5. doi: 10.1007/s00296-008-0677- \\ 6. \\ 4. Duman I, Aydemir K, Tan AK,
}


Dinçer K, Kalyon TA. An unusual case of osteoid osteoma clinically mimicking sacroiliitis. Clin Rheumatol 2007; 26(7): 1158-60. doi: 10.1007/s10067006-0280-8.

5. Maillefert JF, Mischis-Troussard C, Proy A, Piroth C, Tavernier C. Pyomyositis of the psoas and the iliacus muscles mimicking infective sacroiliitis. $\boldsymbol{J}$ Clin Rheumatol 1996; 2(4):231. doi: 10.1097/00124743-19960800000013 .

6. Humphrey SM, Inman RD Metastatic adenocarcinoma mimicking unilateral sacroiliitis. J Rheumatol 1995; 22(5): 970-2.

7. Olivieri I, Gemignani G,
Camerini E, Semeria R, Christou C, Giustarini S, et al. Differential diagnosis between osteitis condensans ilii and sacroiliitis. $\boldsymbol{J}$ Rheumatol 1990; 17(11): 150412.

8. Solmaz D, Onen F, Balci A, Akar S. Pelvic Ewing sarcoma mimicking sacroiliitis. Arthritis Rheum 2013; 65(1): 290. doi: 10.1002/art.37710.

9. Garip Y, Dedeoglu M, Bodur H. Osteomalacia mimicking spondyloarthropathy: a case report. Osteoporos Int 2014; 25(7): $\quad 1983-5$. doi: 10.1007/s00198-014-2716-3.

10. Modaressi K, Fuchs B, Sutter R, Bode P, Meili S, Weber U.
Clinical images: osteoblastoma of the ilium mimicking sacroiliitis. Arthritis Rheum 2013; 65(6): 1674. doi: 10.1002/art.37915.

11. Tezcan ME, Temizkan S, Ozal ST, Gul D, Aydin K, Ozderya A, et al. Evaluation of acute and chronic MRI features of sacroiliitis in asymptomatic primary hyperparathyroid patients. Clin Rheumatol 2016; 35(11): 2777-82. doi: 10.1007/s10067-016-3172-6.

12. Wu K, Hou SM, Huang TS, Yang RS. Thyroid carcinoma with bone metastases: a prognostic factor study. Clin Med Oncol 2008; 2: 129-34. 